\title{
Structure-property relationships of polypropylene-based nanocomposites obtained by dispersing mesoporous silica into hydroxyl-functionalized polypropylene. Part 2: Matrix-filler interactions and pore filling of mesoporous silica characterized by evolved gas analysis
}

\author{
Ryota Watanabe $\mathbb{D}^{1} \cdot$ Hideaki Hagihara $^{1} \cdot$ Hiroaki Sato $^{1}$
}

Received: 13 March 2018 / Revised: 20 April 2018 / Accepted: 22 May 2018 / Published online: 4 July 2018

(c) The Author(s) 2018. This article is published with open access

\begin{abstract}
Nanocomposites containing mesoporous silica (MPS) materials with various pore structures (SBA-15 and MCM-41 types) melt mixed into polypropylene (PP) or PP functionalized with hydroxyl groups (PPOH) were characterized by analytical pyrolysis techniques, such as evolved gas analysis (EGA)-mass spectrometry (MS) and heart-cut EGA-gas chromatography (GC)/MS, to evaluate the interactions between the polymer matrix and MPS and the pore filling of the MPS in the nanocomposite. The EGA-MS measurements revealed that nanocomposites with MPSs evolve specific degradation products, which can be attributed to strong interactions between the polymer molecules and the internal pores. The amount of these specific products increased upon increasing the pore size of the MPS and the hydroxyl content in the polymer matrix. Sufficiently large pores of MPS and high hydroxyl contents in the matrix appear to provide strong interactions because the MPS pores are well-filled with polymer molecules, which contributes to the improved physical properties of the nanocomposites.
\end{abstract}

\section{Introduction}

Characterization of the interface between a polymer matrix and a filler compound in polymer composites has received increasing attention from materials science researchers, as the interfacial structures and properties are believed to govern the performances of these composites [1-7]. However, characterization of these interfaces remains unexplored due to the many barriers to molecular-level analysis

Electronic supplementary material The online version of this article (https://doi.org/10.1038/s41428-018-0096-9) contains supplementary material, which is available to authorized users.

Ryota Watanabe

r.watanabe@aist.go.jp

$\bowtie$ Hiroaki Sato

sato-hiroaki@aist.go.jp

1 Research Institute for Sustainable Chemistry, National Institute of Advanced Industrial Science and Technology (AIST), 1-1-1 Higashi, Tsukuba 305-8565, Japan of interfacial structures. These interfaces have generally been evaluated by speculating about the observations from electron microscopy and examining the physical properties of the materials [8-10]. To better understand the origin of the performances of the composites, there is a compelling need to develop a technique for characterizing the interfaces between the components of the composites. This has encouraged us to explore new characterization techniques, including our previously reported near-infrared spectroscopy method combined with 2D correlation analysis, to examine the adhesion states of the interfaces in polymer nanocomposites. [11].

In Part 1 of this study [12], we fabricated polypropylene (PP)-based nanocomposites with mesoporous silica (MPS) as a filler, and the resultant composites demonstrate good mechanical properties and optical transparency. It is likely that the nanocomposite's properties are strongly affected by the pore-filled state of the MPS. Therefore, a characterization technique that can provide not only information about the interfacial interactions but also molecular-level details of the polymer molecules confined in the pores is needed. In this study, we propose to reveal the structure-property 
relationships in these nanocomposites using evolved gas analysis (EGA) combined with mass spectrometry (MS) or gas chromatography (GC)/MS

These EGA techniques can provide information about molecular-level interactions at the phase interfaces of blended systems such as chitin-based polymer hybrids [13, 14] and nylon 6-clay hybrids [15]. For nanocomposites containing MPSs, the degradation behaviors of polymer molecules confined in the MPS pores may be different from those seen with other polymer molecules due to the strong interaction between the polymer molecules and the pore surfaces. EGA techniques can therefore be useful for analyzing the pore-filling states of MPSs in the nanocomposites, as well as the matrix-filler interactions within the nanocomposites.

In this paper, we studied the degradation behaviors of nanocomposites composed of MPSs with various pore structures (SBA-15-L, SBA-15-S, and MCM-41) and PP or PP functionalized with 1.3 and $6.4 \mathrm{~mol} \%$ hydroxyl groups (PPOH1.3 and PPOH6.4, respectively) using EGA techniques. The pyrolysis products of the polymer matrices in the nanocomposites were monitored directly as a function of temperature using MS. The individual products evolved during the EGA process were characterized in detail using EGA-GC/MS. The effects on the degradation behavior of the interfacial interactions between the matrix and MPS were investigated. The pore-filled state was further elucidated by evaluating the changes in the degradation behavior that could be attributed to the interfacial interactions.

\section{Experimental}

\section{Sample preparation}

The samples used in this study are the same as those described in Part 1 [12]. PPOH synthesized with a 5-hexen1-ol comonomer, containing either $1.3 \mathrm{~mol} \%$ (PPOH1.3; $\left.M_{\mathrm{w}}=503,000\right)$ or $6.4 \mathrm{~mol} \%$ comonomer $\left(\mathrm{PPOH} 6.4 ; M_{\mathrm{w}}=\right.$ $146,000)$, obtained using a previously reported method [1619], or PP pellets (Novatec ${ }^{\circledR}$ MA3, weight-average molecular weight $M_{\mathrm{w}}=397,000$ ) were used as the polymer matrices. The chemical structures of the polymers are shown in Figure S1. Mesoporous SBA-15-L, SBA-15-S, and MCM-41 were purchased from Sigma-Aldrich and used as fillers. The main difference between the MPSs is their pore sizes. The pore sizes of SBA-15-L, SBA-15-S, and MCM-41 are 8.0, 4.4, and $2.9 \mathrm{~nm}$, respectively. As a control experiment, non-porous silica nanospheres (SNSs) with a size of $103 \pm 4 \mathrm{~nm}$ were synthesized using a previously reported method [20] and were also used as filler. The detailed physicochemical properties of the MPS and SNSs were shown in Part 1 of this study [12].
The nanocomposites were prepared by melt mixing the polymer matrices and the fillers using a Labo-Plastmill kneading machine (Toyo Seiki Seisakusho, Tokyo, Japan) at $180{ }^{\circ} \mathrm{C}$ and $60 \mathrm{rpm}$ for $5 \mathrm{~min}$. The same polymer samples without additional nanofillers were also melt mixed to ensure a similar thermal history. The total amount of polymer material, including the filler, was $3 \mathrm{~g}$ during each process. For the structural analyses and mechanical testing, sample sheets $(50 \times 50 \times 0.5 \mathrm{~mm})$ were prepared by hot pressing the samples at $180^{\circ} \mathrm{C}$.

\section{Tensile tests}

Tensile tests were carried out using a multi-purpose stretching tester (EZ-L, Shimadzu, Kyoto, Japan) according to ISO 527-1 using dumbbell-shaped specimens at a crosshead speed of $10 \mathrm{~mm} / \mathrm{min}$ at room temperature. Three specimens were tested for each sample. The area under the stress-strain curve was used to calculate the tensile toughness.

\section{Thermogravimetry (TG) measurements}

Thermogravimetry measurements were performed using an EXSTAR 6000 TG/DTA 6200 (Hitachi High-Tech Science Corporation, Tokyo, Japan). A given sample (approximately $10 \mathrm{mg}$ ) was placed in an aluminum pan and heated from 30 to $510^{\circ} \mathrm{C}$ at a heating rate of $10^{\circ} \mathrm{C} / \mathrm{min}$ under a nitrogen atmosphere.

\section{EGA measurements}

The EGA-MS system used in this study is basically the same as that used in previous reports [13-15, 21-23]. A temperature-programmable micro-furnace pyrolyzer (PY2010D, Frontier Lab, Koriyama, Japan) was directly coupled with a GC/MS system (6890 GC+5973 MS, Agilent Technologies, California, USA). A sample of approximately $0.2 \mathrm{mg}$ was used for the EGA-MS measurements, a quantity small enough to achieve instant thermodynamic equilibrium during programmed heating. A given sample was placed in a deactivated stainless steel sample cup and heated in the pyrolyzer from 60 to $700{ }^{\circ} \mathrm{C}$ at a heating rate of $10^{\circ} \mathrm{C} / \mathrm{min}$ in a helium atmosphere with a flow rate of $50 \mathrm{~mL} /$ min. A proportion of the flow $(1 \mathrm{~mL} / \mathrm{min})$, reduced by a GC splitter (50:1), was continuously introduced into the MS via a transfer capillary (UADTM-5M, $0.25 \mathrm{~mm}$ i.d. $\times 5 \mathrm{~m}$ long, Frontier Lab). The transfer capillary was maintained at 280 ${ }^{\circ} \mathrm{C}$ in the $\mathrm{GC}$ oven to prevent condensation of less volatile products in the capillary. For the MS measurements, electro-ionization (EI) was carried out at $70 \mathrm{eV}$ with an operating mass range of $m / z, 10-450$ and a scan rate of $0.3 \mathrm{~s} /$ scan. 
Heart-cut EGA-GC/MS measurements were performed to identify and quantify the individually evolved products. In this case, the capillary transfer line for the EGA-MS system was replaced by a metal capillary separation column (Ultra Alloy ${ }^{+}-5,0.25 \mathrm{~mm}$ i.d. $\times 5 \mathrm{~m}$ long, Frontier Lab) coated with a $0.25 \mu \mathrm{m}$ film of immobilized $5 \%$ diphenyl95\% dimethylpolysiloxane. The thermal degradation products evolved between 400 and $475^{\circ} \mathrm{C}$ and 475 and $550{ }^{\circ} \mathrm{C}$ were separately trapped at the entrance of the GC column and held at $-196{ }^{\circ} \mathrm{C}$ using a microjet cryotrap (MHT-1030E, Frontier Lab). The formed volatiles from all subjacent temperature levels were redirected by the split vent of the selective sampler (SS-1010E, Frontier Lab). After finishing each heating period, the column temperature was quickly raised to $40{ }^{\circ} \mathrm{C}$, then heated to $320^{\circ} \mathrm{C}$ at a rate of $5^{\circ} \mathrm{C} / \mathrm{min}$ and held for $17 \mathrm{~min}$. The other conditions were basically the same as those used for the EGA-MS measurements described above.

The evolution profiles of the pyrolysis products were observed in total ion current (TIC) and extracted ion monitoring (EIM) modes. The TIC mode represents the added intensities of all mass spectral peaks. In the EIM mode, only a specified mass is extracted from all the mass spectral peaks. The products were identified using the Wiley Mass Spectral Library.

\section{Results and discussion}

\section{Thermal degradation behavior of PPOH6.4 nanocomposites}

Figure 1 displays the TG curves of PPOH6.4 nanocomposites containing $20 \mathrm{wt} \%$ MPSs, such as SBA-15-L, SBA-

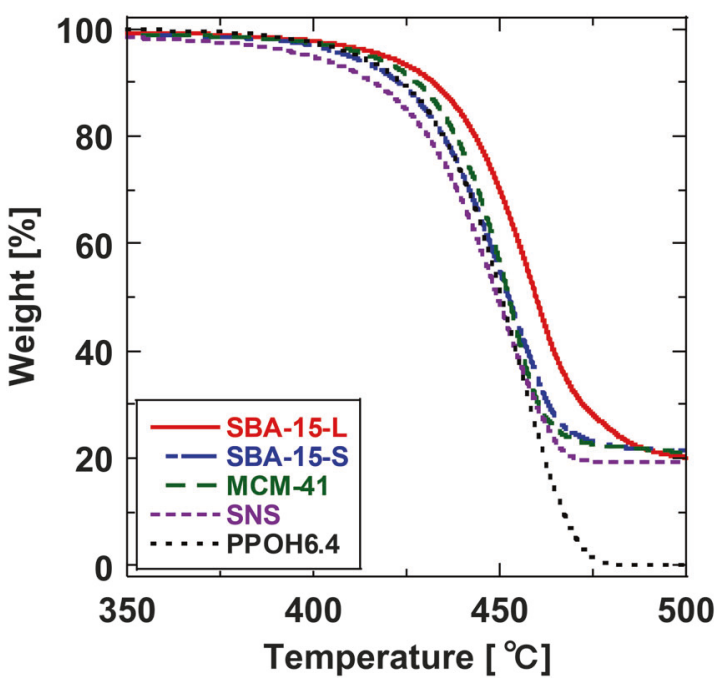

Fig. 1 TG curves of the PPOH6.4 matrix and PPOH6.4 nanocomposites containing $20 \mathrm{wt} \%$ SBA-15-L, SBA-15-S, MCM-41, and SNSs
15-S, and MCM-41, and non-porous SNSs under a nitrogen atmosphere. In each of the TG curves, approximately $100 \%$ of the mass was lost at $500{ }^{\circ} \mathrm{C}$, indicating that all the polymer components are degraded to gas during the heating process. However, the TG analysis gives little chemical information on the degradation products of the matrix and the filler.

For a closer examination of the degradation behaviors of the nanocomposites, EGA, which provides chemical information on the degraded products, was applied to characterize the nanocomposites with MPSs. Figure 2 shows the evolution profiles of the pyrolysis products from the nanocomposite samples observed by EGA-MS in TIC mode, in which the intensities are normalized by the maximum intensity of each TIC curve. Figure $2 \mathrm{a}$ illustrates the TIC curves of nanocomposites containing $20 \mathrm{wt} \%$ MPSs SBA15-L, SBA-15-S and MCM-41, and SNSs. The TIC curves of the PPOH6.4 matrix and its nanocomposite melt mixed with SNSs clearly exhibit single-stage degradation between 400 and $475^{\circ} \mathrm{C}$, peaking near $450{ }^{\circ} \mathrm{C}$ (denoted as Zone A). Interestingly, the second degradation stage at higher temperatures of $475-550{ }^{\circ} \mathrm{C}$ (denoted as Zone B) is further observed for the TIC curves of nanocomposites melt mixed with MPSs. PPOH6.4/SBA-15-L exhibits a notably clear peak reaching its maximum at $485^{\circ} \mathrm{C}$. Figure $2 \mathrm{~b}$ illustrates PPOH6.4 nanocomposites containing 0-30 wt\% SBA-15-L. The intensity in Zone $\mathrm{B}$ can be enhanced by increasing the content of SBA-15-L from 0 to $30 \mathrm{wt} \%$. In addition, the second degradation stage in Zone B is also confirmed by the differential TG curves shown in Figure S3. These observations suggest that the appearance of the second degradation stage is related to the presence of pores and the pore structure of the filler. Hence, the states of the polymer molecules degraded in Zones A and B should be revealed by detailed analyses of the individual degradation products.

\section{Identification of thermal degradation products and possible mechanism of thermal degradation of PPOH6.4/SBA-15-L}

To identify and quantify the thermal degradation products in Zones A and B, heart-cut EGA-GC/MS measurements of the PPOH6.4 and the PPOH6.4 nanocomposites containing $20 \mathrm{wt} \%$ SBA-15-L were performed. Figure 3 shows pyrograms of the pyrolysis products in Zone A $\left(400-475^{\circ} \mathrm{C}\right)$ and Zone $\mathrm{B}\left(475-550^{\circ} \mathrm{C}\right)$. The peak intensities are normalized to $100 \%$ for peak 4 , which is assigned to 2,4dimethyl-1-heptene (a trimer of PP). The assigned products are listed in Table 1.

In the pyrogram for PPOH6.4 (Fig. 3a), C3-C15 hydrocarbons (1-6) derived from the degradation of PPOH6.4 molecules are observed as the major products in a similar manner to the degradation of a typical isotactic PP [24-26]. 
(a)

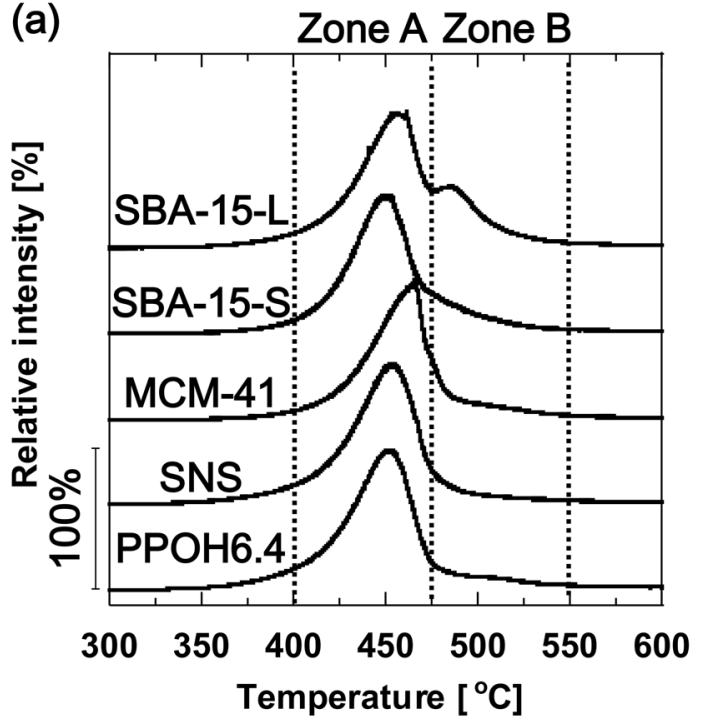

Fig. 2 Evolution profiles of pyrolysis products from a PPOH6.4 matrix and PPOH6.4 nanocomposites containing $20 \mathrm{wt} \%$ of -15-L, SBA-15S, MCM-41, and SNSs and b PPOH6.4 nanocomposites containing 0-30 wt $\%$ SBA-15-L observed by EGA-MS in TIC mode. The two

Furthermore, peaks from alcoholic compounds (7-9), which can be produced by scission of the main chains linked with alcoholic side chains, as shown in Scheme 1, are observed in the pyrogram. The pyrogram for PPOH6.4/ SBA-15-L (Fig. 3b) is quite similar to that for PPOH6.4, indicating that the degradation mode in Zone $\mathrm{A}$ of the PPOH6.4 contained in the PPOH6.4/SBA-15-L nanocomposite is the same as that of pure PPOH6.4 (herein referred to as "common degradation").

The pyrogram for PPOH6.4/SBA-15-L in Zone B appears similar to that in Zone A (Fig. 3c). A close examination of the pyrogram in Zone B, however, reveals that specific peaks are observed in the pyrogram for PPOH6.4/ SBA-15-L in Zone B, especially at a retention time less than $5.5 \mathrm{~min}$ in the area marked by square (i), whereas the peaks from the alcoholic compounds disappear almost entirely in the area marked by square (ii). Thus, in addition to the common degradation of PPOH6.4, a different type of scission of PPOH6.4 occurs in Zone B.

Figure 4 shows an expanded pyrogram of the area marked by the square in Fig. $3 \mathrm{c}$ for retention times of 1.5-5.5 min. Their assignments are listed in Table 1. Note that the specific products evolved in Zone B, which hardly appear in the pyrogram of Zone A shown in Figure S4, are mainly derived from tetrahydrofuran (THF) (10) and unsaturated compounds, such as monoenes $(\mathbf{1 3}, \mathbf{1 7})$, dienes $(11,18)$, cyclic compounds $(12,14,16,19,20,21)$ and aromatics, such as benzene (15) and toluene (22). Although the formation of unsaturated products $(\mathbf{1 3}, \mathbf{1 8})$ is confirmed in the pyrograms of products from PPOH6.4 and PPOH6.4/ SBA-15-L in Zone A, the amounts of observed PPOH6.4/

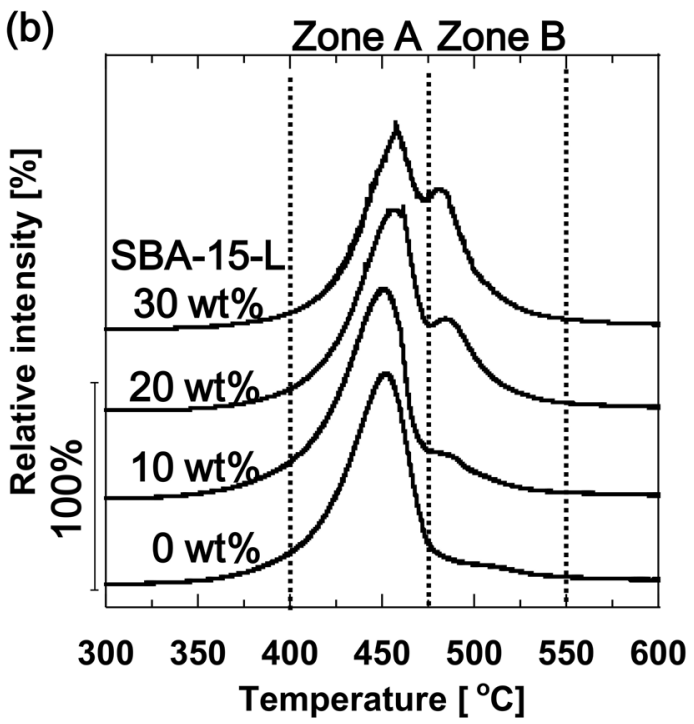

degradation stages at $400-475^{\circ} \mathrm{C}$ and at $475-550^{\circ} \mathrm{C}$ are denoted by Zone $\mathrm{A}$ and Zone $\mathrm{B}$, respectively. Peak intensities are normalized by the maximum intensity of each TIC curve

SBA-15-L products are enhanced in Zone B. The peaks arising from other unsaturated products and THF are not observed in the pyrograms of the products from PPOH6.4 and PPOH6.4/SBA-15-L in Zone A.

The formation of cyclic $(12,14,16,19,20,21)$ and aromatic compounds $(\mathbf{1 5}, \mathbf{2 2})$ in Zone B is likely due to charring reactions promoted by the acidic sites of SBA-15-L [27]. Intimate contact due to the interaction between the polymer molecules and acidic sites is essential for the progression of these charring processes [28]. The components degraded in Zone B are therefore likely to be polymer molecules that have strongly interacted with SBA-15-L. The polymer confined in the pores of SBA-15-L is a possible candidate for the component degraded in Zone B due to trapping of the polymer molecules by multiple adsorption sites inside the pores via hydrogen bonds that are often observed on the interfaces between PPOH and silica [29, 30].

The formation of THF (10) suggests the scission of sidechain branch points of PPOH6.4 in Zone B, as shown in Scheme 2. The evolution of alkene compounds $(\mathbf{1 1}, \mathbf{1 7})$ is presumably due to chain scission of the side-chain branch points of PPOH6.4. The scission of side-chain branch points is likely induced by the interfacial interactions between PPOH6.4 and silica, implying that the PPOH6.4 molecules present in the pores of SBA-15-L are degraded in Zone B.

To study the products containing alcoholic side chains of PPOH6.4 in detail, the evolution behavior of the products was examined in EIM mode at $\mathrm{m} / \mathrm{z} 31$, corresponding to the $\mathrm{CH}_{2} \mathrm{OH}$ ion fragments derived from alcoholic compounds (Fig. 5). Figure 5a shows the TIC curve and EIM curve at $\mathrm{m} / \mathrm{z} 31$ with the intensities normalized by the maximum 


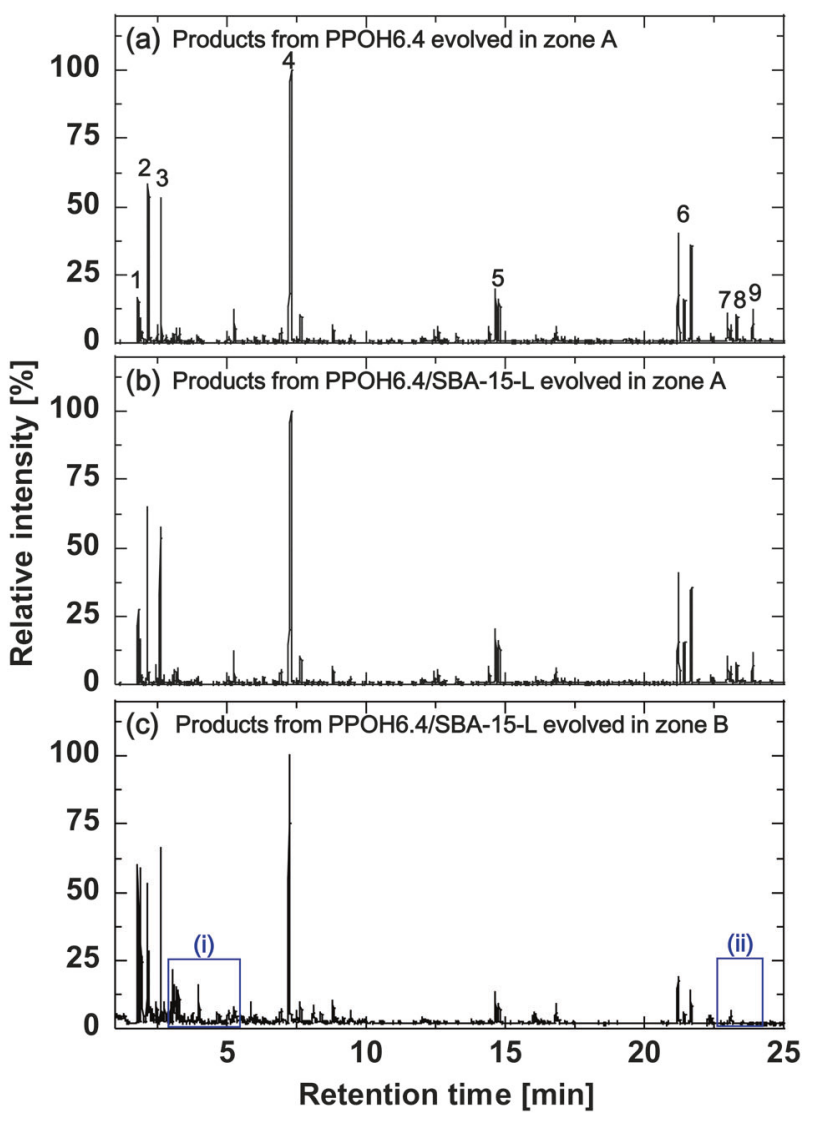

Fig. 3 Pyrograms observed by heart-cut EGA-GC/MS of the pyrolysis products in TIC mode. a Degradation products from PPOH6.4 evolved in Zone A $\left(400-475^{\circ} \mathrm{C}\right)$, and degradation products from PPOH6.4/ SBA-15-L evolved in b Zone A $\left(400-475^{\circ} \mathrm{C}\right)$ and $\mathbf{c}$ Zone B $(475-550$ $\left.{ }^{\circ} \mathrm{C}\right)$. Peak assignments are listed in Table 1 . Peak intensities are normalized to $100 \%$ for peak 4, which is assigned to 2,4-dimethyl-1heptane (trimer). The differences in the pyrograms in $\mathbf{c}$ are marked by squares (i) and (ii)

intensity. The TIC curve exhibits two-stage degradation, although the EIM curve of PPOH6.4/SBA-15-L exhibits single-stage degradation in Zone A. Figure $5 \mathrm{~b}$ shows a pyrogram for PPOH6.4/SBA-15-L in EIM mode $(\mathrm{m} / \mathrm{z} 31)$ in Zone A to identify the alcoholic compounds, which are not observed in the pyrogram in EIM mode $(\mathrm{m} / \mathrm{z} 31)$ in Zone B (Figure S5). The assigned products are listed in Table 1. Alcoholic compounds with various carbon-chain lengths are evolved. Their presence clearly indicates that alcoholic compounds are selectively evolved in Zone A only. They are evolved by degradation of the PPOH6.4 molecules without specific interactions with the pores of SBA-15-L, as these alcoholic compounds are evolved even from pure PPOH6.4 as shown in Fig. 3a. Therefore, it appears that the polymer molecules degraded in Zone A are derived from molecules that are present outside the pores of SBA-15-L. The alcoholic side chains adsorbed on the internal pore walls of SBA-15-L by the interfacial interactions remain in the pores in Zone A. Further heating into Zone B leads to side-
Table 1 Pyrolysis products of PPOH6.4/SBA-15-L according to EGA-GC/MS

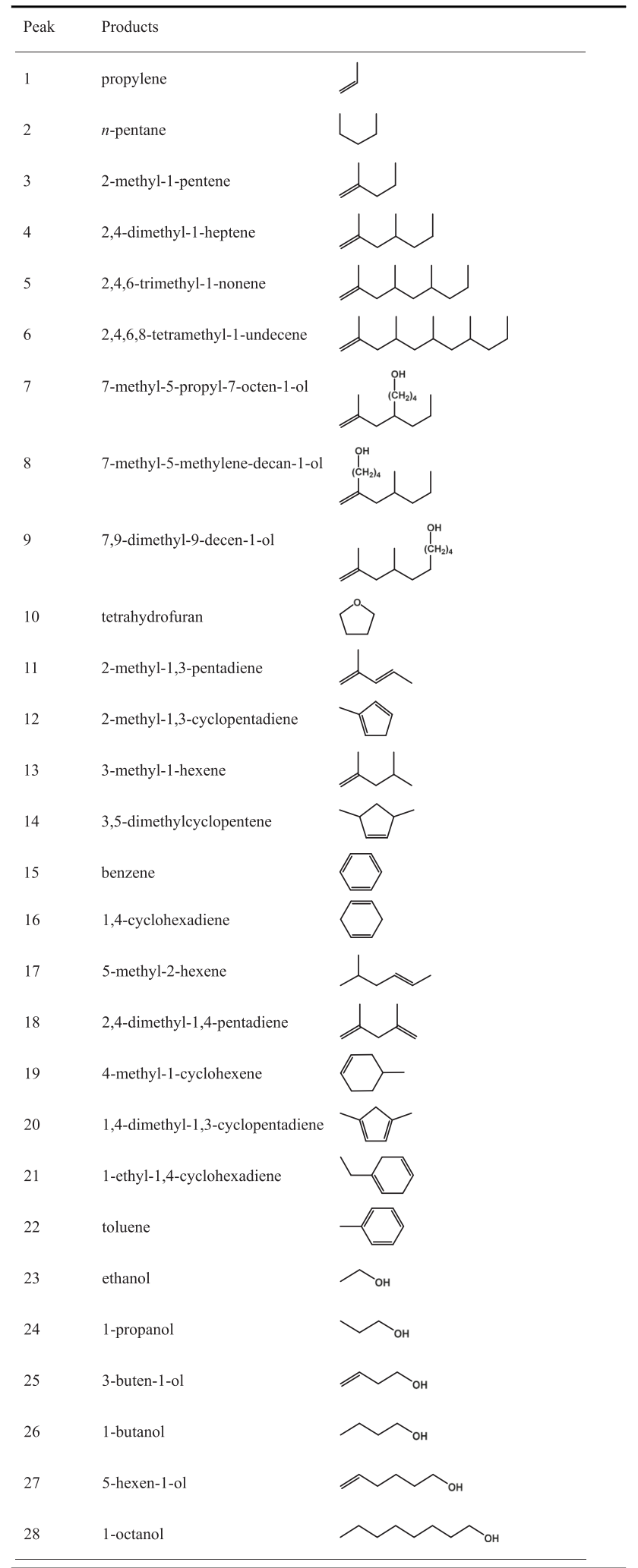

chain scission and cyclization reactions, resulting in the formation of THF (10). 
Scheme 1 The evolution of typical alcoholic products $(\mathbf{8})$

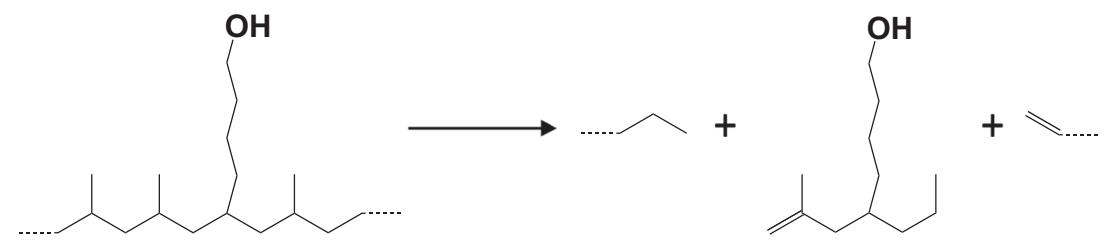

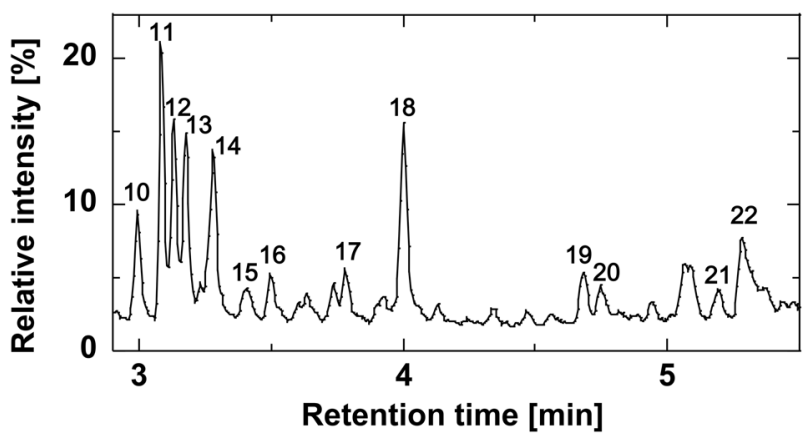

Fig. 4 Expanded pyrogram observed by heart-cut EGA-GC/MS of the pyrolysis products from PPOH6.4/SBA-15-L evolved in Zone B. Peak assignments are listed in Table 1. Peak intensities are normalized to $100 \%$ for the peak assigned to 2,4-dimethyl-1-heptane (a trimer of PP)

Figure 6 illustrates a possible degradation scheme for PPOH6.4/SBA-15-L. In Zone A, the polymer molecules present outside the pores of SBA-15-L are degraded, whereas the polymer molecules that interact with the inside of the SBA-15-L pores remain in the pores and are not degraded. The inhibition of the polymer degradation may be due to radical scavenging by adsorption onto the acidic sites on the silicate surface, which is often observed in typical polymer nanocomposites containing layered silicates [28, 31, 32]. In PPOH6.4/SBA-15-L, the strong interactions of the polymer molecules with the internal pores of SBA-15-L could enhance the radical-scavenging reactions by the acidic sites on the surface of SBA-15-L and inhibit the degradation of PPOH6.4 present in the pores. In the subsequent Zone B degradation, the polymer molecules confined in the pores of SBA-15-L are finally degraded due to insufficient stabilization by radical scavenging at the very high temperatures of Zone B. Here, specific products, such as aromatic, cyclic and alkene compounds, and THF, which are rarely evolved during ordinary degradation, are simultaneously evolved by charring reactions and the elimination of side chains of PPOH6.4 on the silica surface.

\section{Correlation between the MPS structure, MPS pore filling and mechanical properties of the nanocomposites}

In Part 1 of this study, we found that PPOH6.4/SBA-15-L exhibits higher toughness and stiffness than PPOH6.4/
MCM-41, in spite of the smaller relative surface area of SBA-15-L $\left(619 \mathrm{~m}^{2} / \mathrm{g}\right)$ compared to that of MCM-41 (966 $\mathrm{m}^{2} / \mathrm{g}$ ). This implies that the pore-filling structure of MPS in the nanocomposites affects the overall physical properties [12]. According to the analysis in the previous section, the degradation in Zone B is derived from the PPOH6.4 molecules present in the MPS pores. In other words, the pore-filling state of the MPS in the nanocomposites can be evaluated by quantitative spectral measurements of the degradation products in Zone B. This evaluation of the pore-filled state is expected to provide valuable information on how to improve the overall physical properties.

Here, the amount of PPOH6.4 molecules confined in the pores of MPSs with various pore sizes (SBA-15-L, SBA15-S, and MCM-41) was estimated from the intensity observed in Zone B of the TIC curves of the PPOH6.4 nanocomposites, as shown in Fig. 7. The relationship between the pore-filled state of the MPSs and the toughness of the material was also investigated. Figure $7 \mathrm{a}$ illustrates the relationship between the pore size of MPS and the relative peak intensity in Zone B of the TIC curves shown in Fig. 2a. The intensity in Zone B continuously increases with increasing MPS pore size, suggesting that larger pores in MPS enable high penetration of polymer molecules into the pores. The relationship between the normalized intensity in Zone $\mathrm{B}$ and the tensile toughness of the nanocomposites containing MPSs is plotted in Fig. 7b. Interestingly, the tensile toughness tends to continuously improve with increased intensity in Zone B.

Based on these results, we propose a possible mechanism for the observed toughness of PPOH6.4 nanocomposites containing MPSs (Fig. 8). The pores of SBA-15-L are sufficiently large to permit penetration by PPOH6.4 molecules, leading to a high loading of these molecules into the pores. On the other hand, the pores of SBA-15-S and MCM-41 are too small to permit effective pore filling with PPOH6.4. The good pore filling with PPOH6.4/SBA-15-L appears to provide strong anchoring for the polymer molecules to the pore surfaces and restricts the interfacial bond breaking between the matrix and filler, resulting in enhanced toughness. The improved interfacial adhesion due to good pore filling of SBA-15-L may also contribute to the significant improvement in its stiffness and the excellent optical transparency reported in Part 1 of this study [12]. 
Scheme 2 Formation of THF and alkene compounds by the scission of side-chain branch points in Zone B

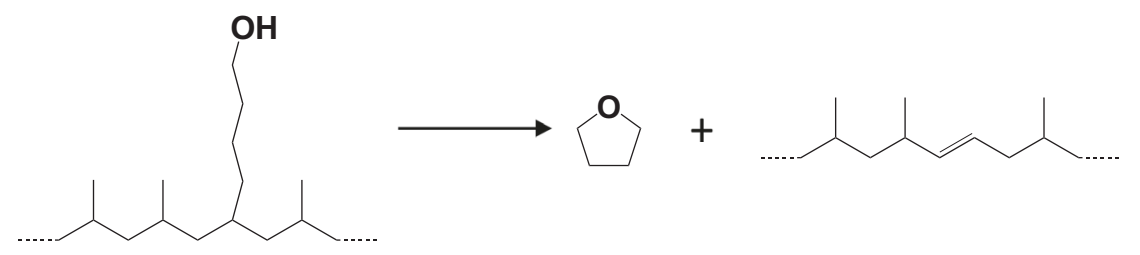

(a)

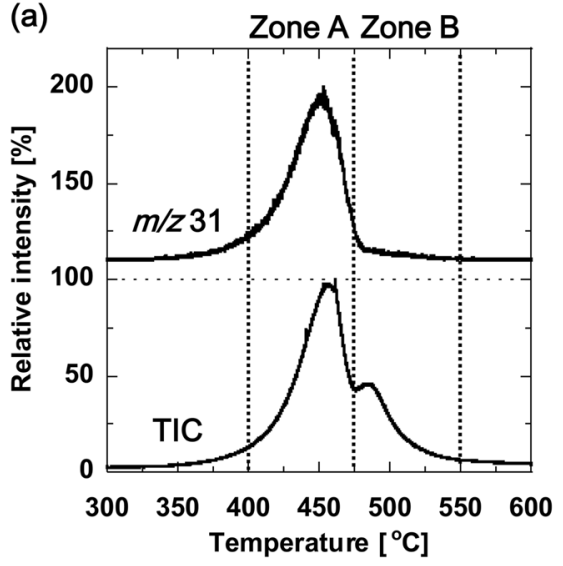

Fig. 5 Investigation in EIM mode of the evolution behavior of products containing the alcoholic side chain of PPOH6.4. a TIC and EIM curves of PPOH6.4/SBA-15-L, and b EIM pyrogram obtained by heart-cut EGA-GC/MS of the pyrolysis products from PPOH6.4/SBA$15-\mathrm{L}$ evolved in Zone A. The ions observed at $\mathrm{m} / \mathrm{z}, 31$ correspond to

(b) $m / z 31$

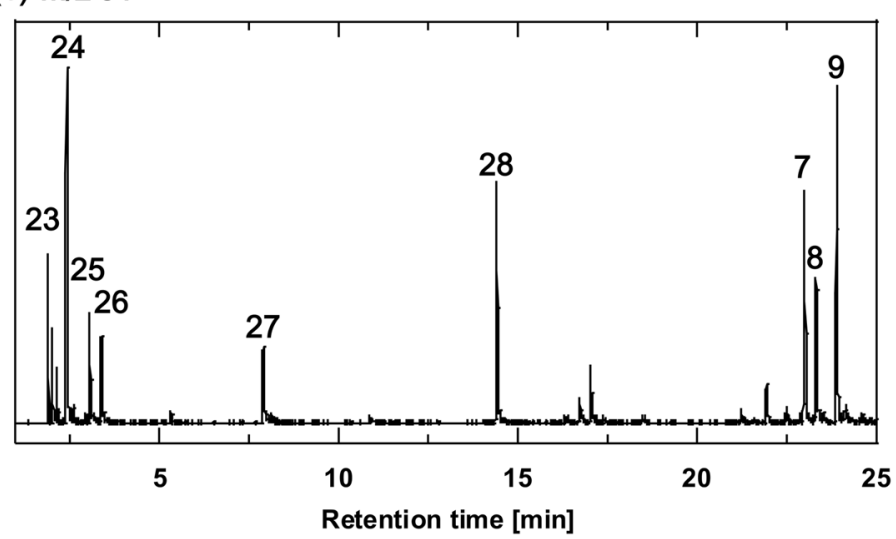

ion fragments of alcoholic compounds. The intensities of the TIC and EIM curves are normalized by the maximum intensity. Peak assignments are listed in Table 1. The content of SBA-15-L in the present composite is $20 \mathrm{wt} \%$
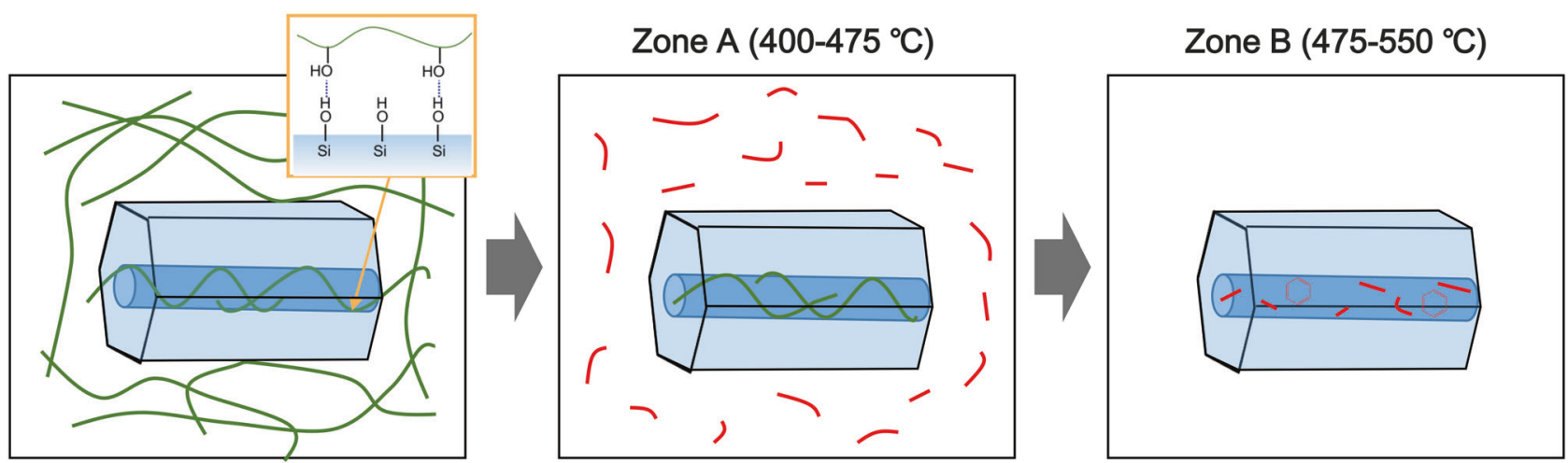

Fig. 6 A schematic illustration of the thermal degradation behavior of PPOH6.4/SBA-15-L

\section{Effects of hydroxyl content in the matrix on the interfacial interactions and toughness of the nanocomposites}

The results of Part 1 of this study indicate that increasing the hydroxyl content in the matrix leads to improved toughness for nanocomposites containing SBA-15-L [12]. It is assumed that the interfacial interactions between the matrix and internal pores of SBA-15-L influence the toughness of the nanocomposites. For a better understanding of the toughening effects with respect to the interfacial interactions, we investigated the intensities of the
TIC curves of the products in Zone B from nanocomposites composed of SBA-15-L and a polymer matrix while varying the amount of hydroxyl groups.

The amount of each polymer matrix (PP, PPOH1.3 or PPOH6.4) confined in the pores of SBA-15-L was examined, and the relationship between the pore-filled state of the SBA-15-L and the toughness was further discussed and is shown in Fig. 9. Figure 9a shows the relationship between the hydroxyl content of the polymer matrix and the relative intensity of the TIC curves in Zone B for PP, PPOH1.3 and PPOH6.4 melt mixed with SBA-15-L (Figure S6). The relative peak intensity in Zone $\mathrm{B}$ is estimated from the 
(a)

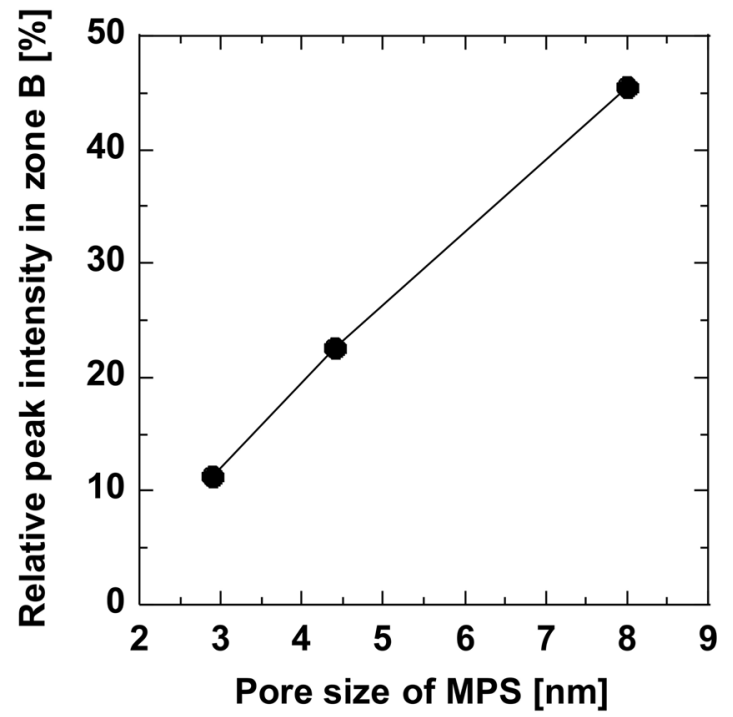

Fig. 7 Evaluation of the pore-filling structure of PPOH6.4 nanocomposites containing SBA-15-L, SBA-15-S, and MCM-41, and the relationship between the pore-filling structure and tensile toughness of the nanocomposites. a The relationship between the pore size of MPS (b)

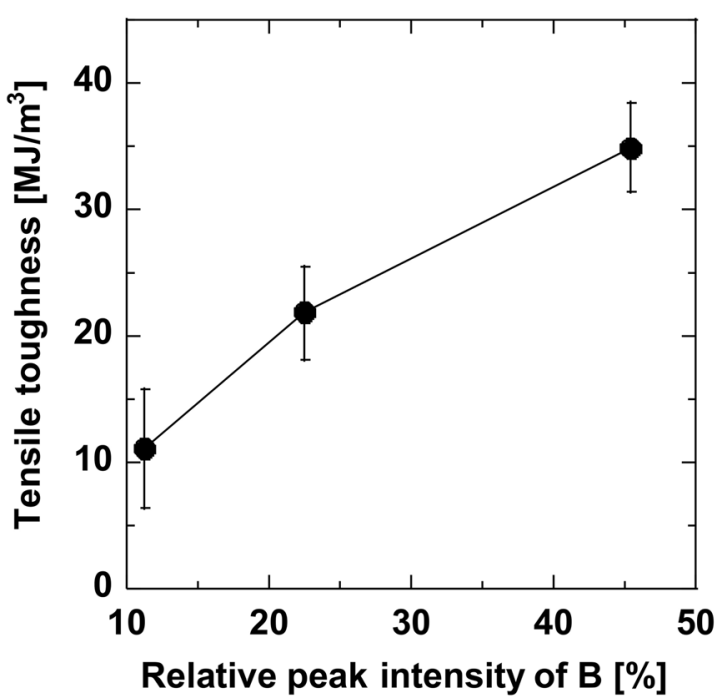

and the relative peak intensity in Zone B of the TIC curves shown in Fig. 2a. b Relationship between the relative peak intensity in Zone B of the TIC curve and the tensile toughness. The content of fillers in the present composites is $20 \mathrm{wt} \%$
Fig. 8 A schematic illustration of the correlation between the pore-filling structure and toughness of the PPOH6.4 nanocomposites containing MPSs (a) SBA-15-L (large pore)

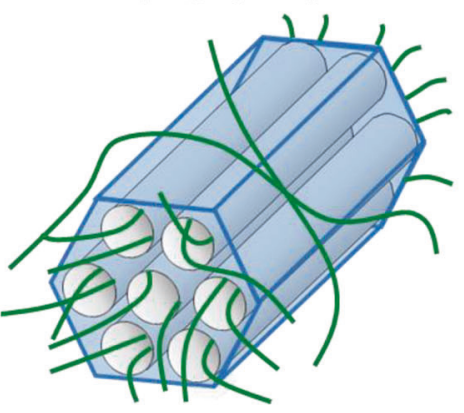

High loading

(b) SBA-15-S and MCM-41 (small pore)

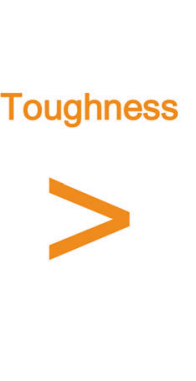

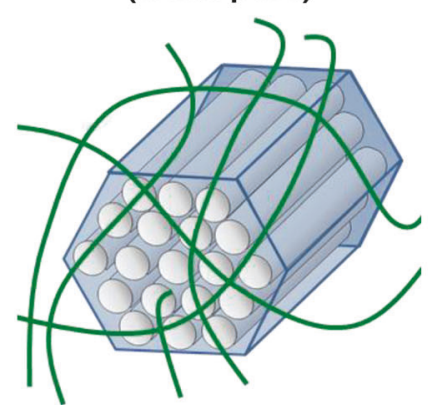

Low loading maximum intensity of the peak or the shoulder peak in Zone B. The intensity in Zone B is continuously increased by increasing the hydroxyl content in the matrix, implying that the interaction between the matrix and the pores of SBA-15$\mathrm{L}$ is enhanced by increasing the number of hydroxyl groups in the matrix. Furthermore, the relationship between the intensity in Zone B and the tensile toughness of the nanocomposites composed of SBA-15-L and PP, PPOH1.3 or PPOH6.4 is plotted in Fig. 9b. This clearly indicates that an increase in intensity in Zone B indicates improved tensile toughness, possibly due to efficient pore filling of SBA-15$\mathrm{L}$ and the matrix - filler interactions.

Figure 10 is a schematic illustration of the interactions between the polymer molecules and the pore surfaces in the nanocomposites of $\mathrm{PPOH}$ melt mixed with SBA-15-L. The incorporation of hydroxyl groups into the polymer molecules plays a key role in the pore filling of SBA-15-L, wherein the polymer undergoes interfacial interactions with the pores of SBA-15-L via hydrogen bonding. This provides evidence that the good pore filling and high interfacial interactions result from the incorporation of hydroxyl groups into the polymer molecules and are essential for improved toughness in the nanocomposites.

\section{Conclusions}

The matrix-filler interactions and pore-filled state of the MPSs in the nanocomposites of PP, PPOH1.3, and PPOH6.4 melt mixed with MPSs (SBA-15-L, SBA-15-S, 
(a)

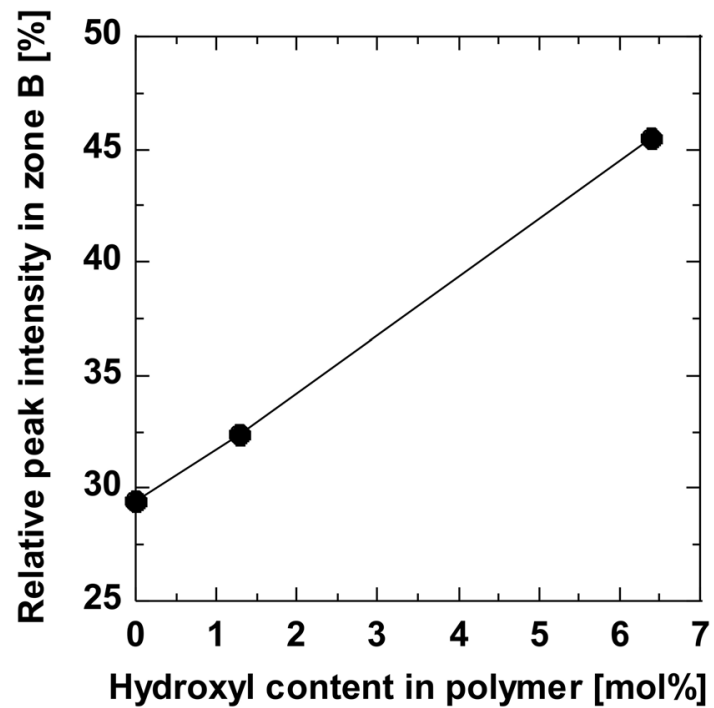

Fig. 9 Evaluation of the pore-filling structures of PP, PPOH1.3, and PPOH6.4 containing $20 \mathrm{wt} \%$ SBA-15-L, and the relationship between the pore-filled structure and tensile toughness of the resultant nanocomposites. a Relationship between the hydroxyl content in the (b)

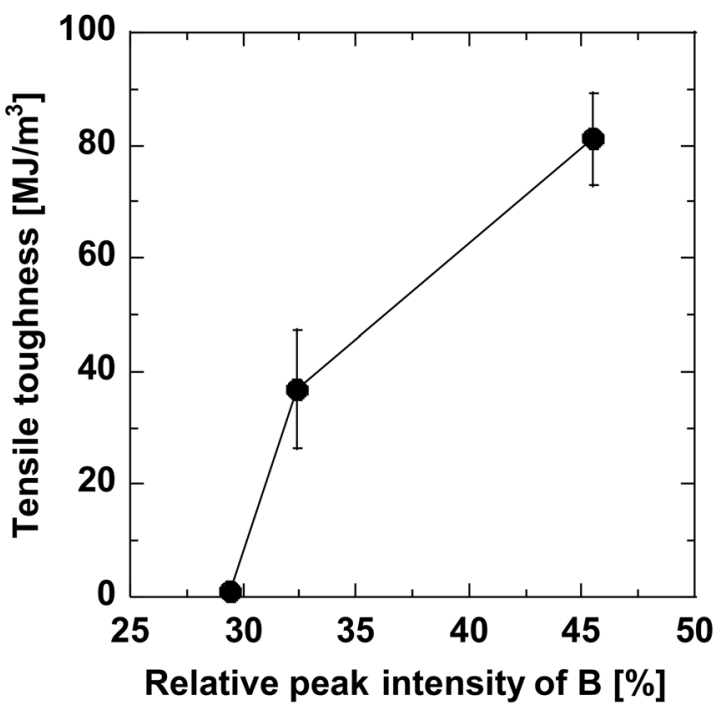

polymer and the relative peak intensity in Zone B of the TIC curves shown in Figure S6. b Relationship between the relative peak intensity in Zone B and the tensile toughness. These graphs represent nanocomposites containing $10 \mathrm{wt} \%$ SBA- $15-\mathrm{L}$

$400-475{ }^{\circ} \mathrm{C}$ (Zone A) arising from the common degradation of the polymer and high-temperature degradation at $475-550{ }^{\circ} \mathrm{C}$ (Zone B) arising from the degradation of polymer molecules strongly interacting with the internal pore surfaces of MPS. PPOH6.4/SBA-15-L evolved greater amounts of products in Zone B compared to PPOH6.4/ SBA-15-S and PPOH6.4/MCM-41, suggesting the best pore filling for PPOH6.4/SBA-15-L, possibly because the pores in SBA-15-L are sufficiently large to allow penetration by the PPOH6.4 molecules. The good pore filling of SBA-15-L provides improved mechanical properties, such as toughness and stiffness, and very good optical transparency. The thermal degradation behaviors of the nanocomposites composed of SBA-15-L and PP, PPOH1.3 or PPOH6.4 were further examined to elucidate the effects of the hydroxyl content in the matrix on the interactions between the polymer molecules and the internal pore surfaces. An increased hydroxyl group content in the polymer molecules resulted in an enhancement in the interactions that contribute to improved matrix - filler adhesion, leading to improved toughness. Thus, the observed thermal behaviors provided chemical information about the matrix - filler interactions and the pore-filled state of the MPS, which are closely related to the physical properties of the nanocomposites containing MPSs. We believe that our findings will contribute to further improvements in the properties of new polymer nanocomposites.

Acknowledgements This work was financially supported by the Izumi Science and Technology Foundation. 


\section{Compliance with ethical standards}

Conflict of interest The authors declare that they have no conflict of interest.

Open Access This article is licensed under a Creative Commons Attribution 4.0 International License, which permits use, sharing, adaptation, distribution and reproduction in any medium or format, as long as you give appropriate credit to the original author(s) and the source, provide a link to the Creative Commons license, and indicate if changes were made. The images or other third party material in this article are included in the article's Creative Commons license, unless indicated otherwise in a credit line to the material. If material is not included in the article's Creative Commons license and your intended use is not permitted by statutory regulation or exceeds the permitted use, you will need to obtain permission directly from the copyright holder. To view a copy of this license, visit http://creativecommons. org/licenses/by/4.0/.

\section{References}

1. Zou H, Wu S, Shen J. Polymer/silica nanocomposites: preparation, characterization, properties, and applications. Chem Rev. 2008;108:3893-957.

2. Zhou TH, Ruan WH, Rong MZ, Zhang MQ, Mai YL. Keys to toughning of non-layered nanoparticles/polymer composites. Adv Mater. 2007;19:2667-71.

3. Tjong SC. Structural and mechanical properties of polymer nanocomposites. Mater Sci Eng R. 2006;53:73-197.

4. Taniike T, Toyonaga M, Terano M. Polypropylene-grafted nanoparticles as a promising strategy for boosting physical properties of polypropylene-based nanocomposites. Polym (Guildf). 2014;55:1012-9.

5. Bikiaris DN, Papageorgiou GZ, Pavlidou E, Vouroutzis N, Palatzoglou P, Karayannidis GP. Preparation by melt mixing and characterization of isotactic polypropylene/ $\mathrm{SiO}_{2}$ nanocomposites containing untreated and surface-treated nanoparticles. J Appl Polym Sci. 2006;100:2684-96.

6. Fu SY, Feng XQ, Lauke B, Mai YW. Effects of paricle size, particle/matrix interface adhesion and particle loading on mechanical properties of particulate-polymer composites. Compos Part B Eng. 2008;39:933-61.

7. Bikiaris D, Vassiliou A, Chrissafis K, Paraskevopoulos KM, Jannakoudakis A, Docoslis A. Effect of acid treated multi-walled carbon nanotubes on the mechanical, permeability, thermal properties and thermo-oxidative stability of isotactic polypropylene. Polym Degrad Stab. 2008;93:952-67.

8. Sgriccia N, Hawley MC, Misra M. Characterization of natural fiber surfaces and natural fiber composites. Compos Part A Appl Sci Manuf. 2008;39:1632-7.

9. Alemdar A, Sain M. Biocomposites from wheat straw nanofibers: morphology, thermal and mechanical properties. Compos Sci Technol. 2008;68:557-65.

10. Arbelaiz A, Fernández B, Cantero G, Llano-Ponte R, Valea A, Mondragon I. Mechanical properties of flax fibre/polypropylene composites. Influence of fibre/matrix modification and glass fibre hybridization. Compos Part A Appl Sci Manuf. 2005;36: 1637-44.

11. Watanabe R, Shinzawa H, Kunioka M, Mizukado J, Suda H, Hagihara H. Reinforcement mechanism of functionalized polypropylene containing hydroxyl group nanocomposites studied by rheo-optical near-infrared spectroscopy. Eur Polym J. 2017;92: $86-96$.
12. Watanabe R, Hagihara $H$, Sato $H$. Structure-property relationships of polypropylene-based nanocomposites obtained by dispersing mesoporous silica into hydroxyl-functionalized polypropylene. Part 1: toughness, stiffness and transparency. Polym J. 2018. https://doi.org/10.1038/s41428-018-0095-x

13. Sato H, Tsuge S, Ohtani H, Aoi K, Takasu A, Okada M. Characterization of chitin-based polymer hybrids by temperatureprogrammed analytical pyrolysis techniques. 1. Chitin-graft-poly (2-methyl-2oxazoline)/poly(vinyl chloride) blends. Macromolecules. 1997;30:4030-7.

14. Sato H, Ohtani H, Tsuge S, Aoi K, Takasu A, Okada M. Characterization of chitin-based polymer hybrids by temperatureprogrammed analytical pyrolysis techniques. 2. Chitin-graft-poly (2-methyl-2-oxazoline)/poly(vinyl alcohol) brends. Macromolecules. 2000;33:357-62.

15. Sato H, Ohtani H, Harada R, Tsuge S, Kato M, Usuki A. Polymer/ silicate interaction in nylon 6-clay hybrid studied by temperature programmed pyrolysis techniques. Polym J. 2006;38:171-7.

16. Hagihara $\mathrm{H}$, Ishihara T, Hoang TB, Shiono T. Precise control of microstructure of functionalized polypropylene synthesized by the ansa-zirconocene/MAOcatalysts. J Polym Sci Part A Polym Chem. 2008;46:1738-48.

17. Iizuka $Y$, Sugiyama J, Hagihara H. Unexpected mechanical properties of functionalized polypropylene: tensile test, Charpy impact tensile test, DSC, and WAXD analysis of poly(5 - hexen1-ol-co-propylene). Macromolecules. 2009;42:2321-3.

18. Hagihara H, Ito K, Kimata S. Comprehensive study of altered amorphous structure in functionalized polypropylenes exhibiting high tensile strength. Macromolecules. 2013;46:4432-7.

19. Hagihara H, Tsuchihara K, Takeuchi K, Murata M, Ozaki H, Shiono T. Copolymerization of ethylene or propylene with $\alpha$-olefins containing hydroxyl groups with zirconocene/methylaluminoxane catalyst. J Polym Sci Part A Polym Chem. 2004;42:52-58.

20. Watanabe R, Yokoi T, Kobayashi E, Otsuka Y, Shimojima A, Okubo T, Tatsumi T. Extension of size of monodisperse silica nanospheres and their well-ordered assembly. J Colloid Interface Sci. 2011;360:1-7.

21. Sato H, Kondo K, Tsuge S, Ohtani H, Sato N. Mechanisms of thermal degradation of a polyester flame-retarded with antimony oxide brominated polycarbonate studied by temperatureprogrammed analytical pyrolysis. Polym Degrad Stabil. 1998;62:41-8

22. Watanabe C, Hosaka A, Kawahara Y, Tobias P, Ohtani H, Tsuge S. GC-MS analysis of heart-cut fractions during evolved gas analysis of polymeric materials. LCGC North Am. 2002;20:374-8.

23. Heigenmoser A, Liebner F, Windeisen E, Richter K. Investigation of thermally treated beech (Fagus sylvatica) and spruce (picea abies) by means of multifunctional analytical pyrolysis-GC/MS. J Anal Appl Pyrolysis. 2013;100:117-26.

24. Tsuge S, Ohtani H, Watanabe C. Pyrolysis-GC/MS data book of synthetic polymers, first ed. Oxford: Elsevier; 2011.

25. Sugimura Y, Nagaya T, Tsuge S, Murata T, Takeda T. Microstructural characterization of polypropylenes by high-resolution pyrolysis-hydrogenation glass capillary gas chromatography. Macromolecules. 1980;13:928-32.

26. Ohtani H, Tsuge S, Ogawa T, Elias H-G. Studies on stereospecific sequence distributions in polypropylenes by pyrolysishydrogenation fused-silica capillary gas chromatography. Macromolecules. 1984;17:465-73.

27. Szczodrowski K, Prélot B, Lantenois S, Douillard J-M, Zajac J. Effect of heteroatom doping on surface acidity and hydrophilicity of Al, Ti, Zr-doped mesoporous SBA-15. Micro Mesop Mater. 2009;124:84-93.

28. Kiliaris P, Papaspyrides CD. Polymer/layered silicate (clay) nanocomposites: an overview of flame retardancy. Prog Polym Sci. 2010;35:902-58. 
29. Watanabe R, Kunioka M, Mizukado J, Suda H, Hagihara H. Highly ductile polypropylene-based nanocomposites by dispersing monodisperse silica nanospheres in functionalized polypropylene containing hydroxyl groups. Polym (Guildf). 2016;99: 63-71.

30. Watanabe R, Kunioka M, Sato H, Mizukado J, Hagihara H. Management of both toughness and stiffness of polypropylene nanocomposites using poly(5-hexen-1-ol-co-propylene) and silica nanospheres. Polym Adv Technol. 2018;1:417-23.

31. Zaikov GE, Lomakin SM. Ecological issue of polymer flame retardancy. J Appl Polym Sci. 2002;86:2449-62.

32. Dasari A, Yu Z-Z, Mai Y-W, Liu S. Flame retardancy of highly filled polyamide 6/clay nanocomposites. Nanotechnology. 2007;18:445602. 